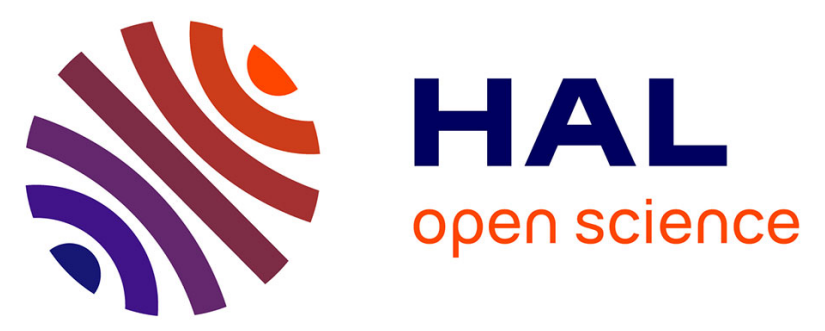

\title{
Multi-scale analysis of the dielectric properties and structure of resin/carbon-black nanocomposites
}

\author{
S. Paciornik, O. da Fonseca Martins Gomes, Arnaud Delarue, Sylvie \\ Schamm-Chardon, Dominique Jeulin, Alain Thorel
}

\section{To cite this version:}

S. Paciornik, O. da Fonseca Martins Gomes, Arnaud Delarue, Sylvie Schamm-Chardon, Dominique Jeulin, et al.. Multi-scale analysis of the dielectric properties and structure of resin/carbon-black nanocomposites. European Physical Journal: Applied Physics, 2003, 21 (1), pp.17-26. 10.1051/epjap:2002107 . hal-00166093

\section{HAL Id: hal-00166093 \\ https://hal.science/hal-00166093}

Submitted on 21 Jun 2019

HAL is a multi-disciplinary open access archive for the deposit and dissemination of scientific research documents, whether they are published or not. The documents may come from teaching and research institutions in France or abroad, or from public or private research centers.
L'archive ouverte pluridisciplinaire HAL, est destinée au dépôt et à la diffusion de documents scientifiques de niveau recherche, publiés ou non, émanant des établissements d'enseignement et de recherche français ou étrangers, des laboratoires publics ou privés. 


\title{
Multi-scale analysis of the dielectric properties and structure of resin/carbon-black nanocomposites
}

\author{
S. Paciornik ${ }^{1,3}$, O. da Fonseca Martins Gomes ${ }^{1}$, A. Delarue ${ }^{2,3}$, S. Schamm ${ }^{4}$, D. Jeulin ${ }^{2}$, and A. Thorel ${ }^{3, \text { a }}$ \\ 1 DCMM PUC-Rio, PO Box 38008, Gávea, Rio de Janeiro, RJ, 22453-900, Brazil \\ 2 Centre de Morphologie Mathématique, École des Mines de Paris, 35 rue Saint-Honoré, 77300 Fontainebleau, France \\ 3 Centre des Matériaux, École des Mines de Paris, BP 87, 91003 Evry Cedex, France \\ 4 CEMES/CNRS, BP 4347, 31055 Toulouse Cedex, France
}

Received: 24 July 2002 / Received in final form: 20 September 2002 / Accepted: 1 October 2002 Published online: 29 November 2002 - (c) EDP Sciences

\begin{abstract}
Dielectric properties of resin/carbon-black nanocomposites were measured and shown to differ from results given by a random sets modeling approach. The origin of the discrepancies was traced back to the presence of sets of carbon planes, detached from the carbon-black particles during the composite preparation. These planes are dispersed in the resin matrix and are nearly invisible, even in HRTEM images. EELS measurements revealed the signature of bonding states typical of graphite-like compounds, in regions of the matrix previously supposed to be free of carbon-black. A new approach to texture characterization of HRTEM images, using Haralick parameters based on pixel intensity co-occurrence matrices, showed strong differences between pure resin and regions of the composite between carbon particles. The nano-scale characterization results explain the values obtained in macroscopic measurements.
\end{abstract}

PACS. 72.80.Tm Composite materials $-61.46 .+\mathrm{w}$ Nanoscale materials: clusters, nanoparticles, nanotubes, and nanocrystals - 68.37.Lp Transmission electron microscopy (TEM)

\section{Introduction}

The concept of composite material is often applied to structural applications. There, the intimate mixing of two or more phases with different mechanical properties gives rise to a material for which the performance is enhanced by a factor of 10 to 100 . But there are functional applications in which this concept, when applied, can lead to a much wider range of property variations, simply because many physical properties - such as electrical conductivity - can vary by many orders of magnitude.

The material under consideration here has such capabilities regarding its dielectric properties. It consists of a polymeric resin in which carbon-black nano-sized particles are dispersed. The resin has a high electrical resistivity whereas the carbon-black is conductive. Then, mixing both materials in appropriate proportions enables precise tailoring of the dielectric properties of the composite, allowing a variation of several orders of magnitude.

At concentrations that allow the carbon-black particles to approach the percolation threshold for conduction it is found that the absorption of electromagnetic waves in the Gigahertz frequency range is most efficient [1]. This can be of uttermost interest in civil applications (mobile phone or building shielding) as well as in military applications (radar shielding, stealth behavior).

\footnotetext{
a e-mail: alain.thorel@mat.ensmp.fr
}

Optimization of the composite properties can be obtained on a scientific basis by coupling TEM observation at moderate magnification and a numerical random set analysis based on a Boolean approach in which the properties of the pure resin and of the carbon-black are introduced [2]. This is a very powerful approach that can be successfully applied to different conditions of elaboration [3] provided that, during the fabrication of the composite, there is no change in the structure and properties of the components and no specific interface-related mechanisms appear. If these conditions are not fulfilled, the model has to be refined and the real properties of the components and interfaces have to be locally evaluated; this is often very difficult to achieve if the property has to be measured at a scale of the order of the coherence length of the microstructure, typically less than one micrometer [4].

To fully understand why properties have locally changed and be able to re-adjust the process, it is also necessary to investigate the material structure at the relevant scale. This is exactly the scope of the present paper in which we show that the bulk dielectric properties of the resin/carbon- black composite under consideration are drastically different from what is expected if the material is simulated by an adequate combination of pure resin and pure carbon. Therefore, a methodology is proposed and a set of tools is presented that allow quantitative investigation of the composite at nanometer scale, in order to give evidence of the changes in the material during processing. 


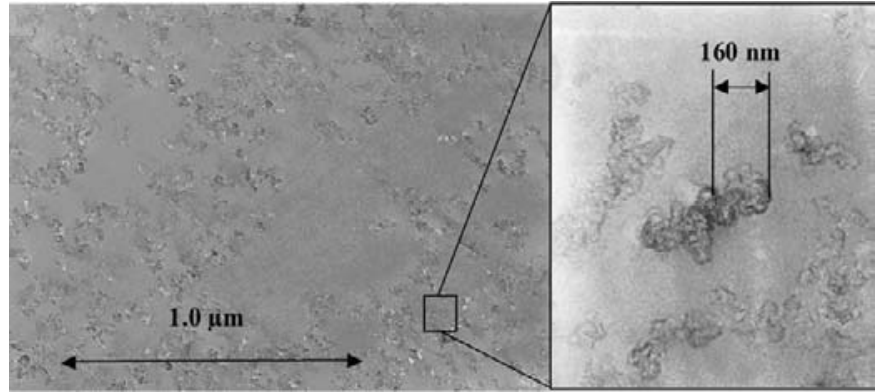

Fig. 1. CTEM image of the HC composite material.

\section{Preliminary mesoscale characterization}

\subsection{Material description}

The composite material was obtained from a liquid resin in which carbon-black particles, 20 to 40 nanometers in size, were dispersed with a propeller. Carbon particles have a tendency to agglomerate in clusters 160 nanometers in size - the cohesion force between these particles is very strong, and the propeller must develop shear forces intense enough to be able to overcome that cohesion force.

The carbon-black particles consist of turbostratic spheres with an onion-like structure the skin of which is made of a stacking of (100) planes with a d-spacing of $0.37 \mathrm{~nm}$. The bonding between theses planes is not very strong since only Van der Waals forces are involved.

Two composite samples were studied, one with lower carbon concentration (LC, $2.64 \mathrm{wt} \%$ ) and one with $30 \%$ higher concentration ( $\mathrm{HC}, 4 \mathrm{wt} \%$ ).

Conventional TEM images at 17000X and 122000X were obtained in a Philips EM430 microscope operating at $300 \mathrm{kV}$. In order to enhance the image contrast, the images were recorded under slightly under focused conditions. Figure 1 shows a typical image for the HC material. The width of the Fresnel fringes around the black carbon clusters were absolutely negligible and did not affect further calculations based on quantitative image analysis.

\subsection{Permittivity measurements}

Macroscopic dielectric permittivity measurements were carried out on disks ( $3 \mathrm{~mm}$ thick and $21 \mathrm{~mm}$ in diameter) of both composite samples. An HP 4291B impedance analyzer was used at $1 \mathrm{GHz}$. The complex permittivity was found to be $\varepsilon^{*} / \varepsilon_{0}=15.0+8.0 \mathrm{i}$ for the LC sample and $\varepsilon^{*} / \varepsilon_{0}=47.7+34.0 \mathrm{i}$ for the $\mathrm{HC}$ sample, where $\varepsilon_{0}$ is the permittivity of vacuum.

Thus, an increase of $30 \%$ in carbon concentration, leads to a drastic increase in the permittivity. Clearly, the composite is strongly affected by the addition of a carbon phase since these values indicate the contribution of a conduction mechanism.

\subsection{Mesoscopic morphological modeling}

Starting from images similar to the micrographs given in Figure 1, it is possible to extract the carbon particles by morphological image segmentation $[1,5]$. Then multi-scale models of random sets were used to describe the arrangement of particles in space $[1,2,5]$. The basic model is the Boolean model introduced by Matheron $[6,7]$. It reproduces overlapping grains in space and is therefore a good candidate for the mesostructure of these nanocomposites. It is obtained in two steps:

- implantation of Poisson points $x_{k}$ with intensity $\theta$ (average number per unit volume) in the space;

- implantation of a random primary grain $A^{\prime}$ on every Poisson point, with possible overlaps between grains.

The material $A$ is obtained by taking the union (noted $\cup$ ) of all the implanted primary grains, according to equation (1):

$$
A=\cup_{x k} A_{x k}^{\prime} .
$$

For this type of model the theoretical expression of the probability for a set $K$ to be included in the complementary set of grains $A^{\mathrm{c}}$, is known. For instance, the covariance of the complementary set of grains, $Q(h)$, is defined and given by

$$
Q(h)=P\left\{x \in A^{\mathrm{c}}, x+h \in A^{\mathrm{c}}\right\}=q^{2} \exp (\theta K(h))=q^{2-r(h)}
$$

where $Q(0)=q\left(\right.$ volume fraction of $\left.A^{\mathrm{c}}\right), K(h)$ is the geometrical covariogram of the grains

$$
K(h)=\bar{V}\left(A^{\prime} \cap A_{-h}^{\prime}\right)
$$

the average volume of the intersection of a random grain with itself translated after a translation of vector $h$, and $r(h)$ is the reduced geometrical covariogram

$$
r(h)=K(h) / K(0) .
$$

The covariance $C(h)$ of the random set $A$ (here the grains) is deduced from the covariance $Q(h)$ by

$$
C(h)=1-2 q+Q(h)
$$

In the present application, populations of spheres with a random diameter were used as primary grains $A^{\prime}$. To account for the superposition of scales, a combination of elementary models was used as follows (see Fig. 2):

- the carbon nanoparticles were described by a first Boolean model of spheres $A_{1}$ (with a diameter equal to $32 \mathrm{~nm}$ and a volume fraction equal to $p_{1}$ );

- aggregates were represented by a second Boolean model of spheres $A_{2}$ (with a diameter equal to $130 \mathrm{~nm}$ and a volume fraction equal to $p_{2}$ );

- zones of resin which look empty of carbon-black (later called a mixed resin) can be simulated by a third Boolean model of spheres $A_{3}$ (with a random diameter following an exponential distribution with an average $d_{3}$ and a volume fraction equal to $q_{3}$ ). 

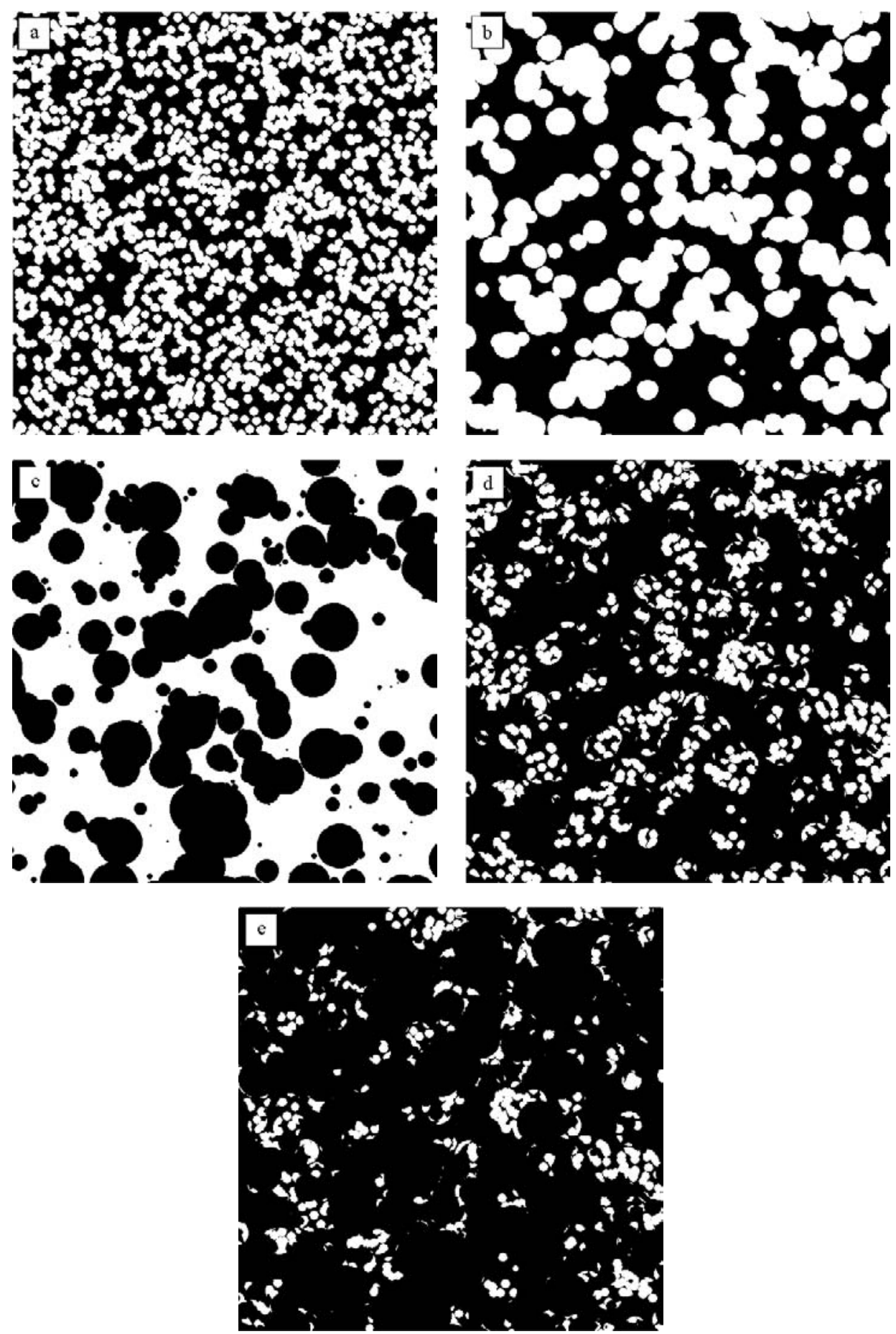

Fig. 2. Multi-scale modelling of the nanocomposites resin/carbon-black. (a) Nanoparticles $\left(A_{1}\right)$. (b) Aggregates $\left(A_{2}\right)$. (c) Resin containing carbon black $\left(\left(A_{3}\right)^{\mathrm{c}}\right)$. (d) $A_{1} \cap A_{2}$. (e) Final structure $A_{1} \cup A_{2} \cup\left(A_{3}\right)^{\mathrm{c}}$.

The final model to describe the carbon phase $A$ was obtained by intersection (noted $\cap$ ) of the three scales

$$
A=A_{1} \cap A_{2} \cap\left(A_{3}\right)^{\mathrm{c}} .
$$

The covariance of the intersection, for three independent random sets $A_{1}, A_{2}$ and $A_{3}$ is given by

$$
C(h)=C_{1}(h) C_{2}(h) Q_{3}(h)
$$

and the volume fraction of carbon is given by

$$
p=C(0)=p_{1} p_{2} q_{3} .
$$

For the identification of the model, the quadratic error between the experimental covariance and the theoretical covariance (with a correction accounting for the thickness of sections [5]) was minimized, and the free parameters $\left(d_{3}, p_{2}, q_{3}\right)$ were estimated. For the LC composite (with 

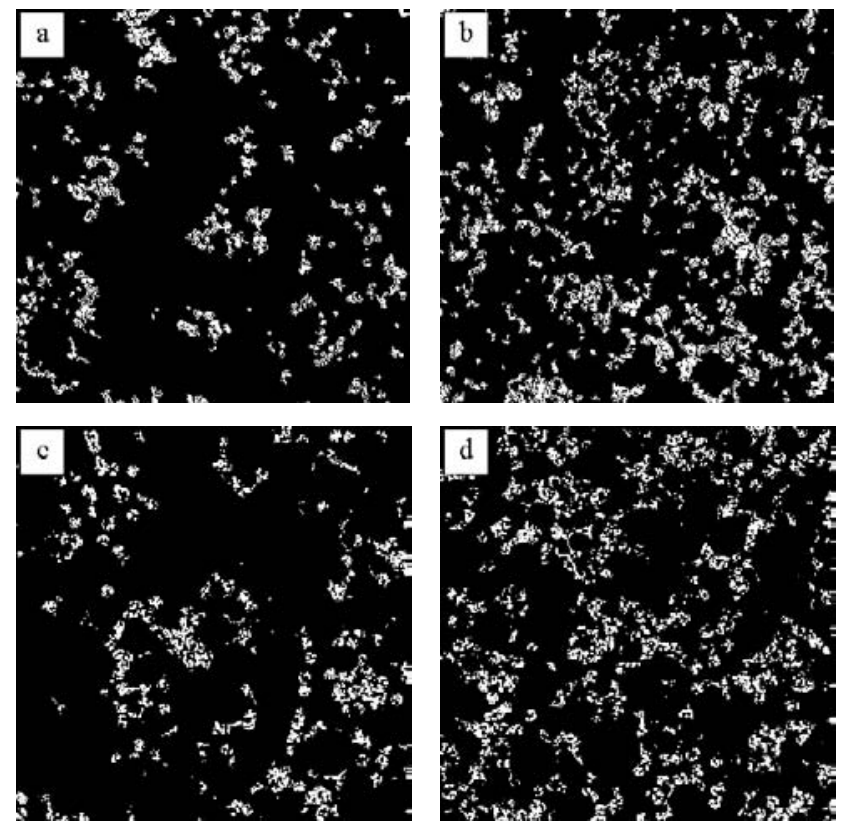

Fig. 3. Comparison between segmented TEM images $(1.5 \times$ $\left.1.5 \mu \mathrm{m}^{2}\right)$ and simulated images after identification of the parameters of a multi-scale random set model for two materials. (a, b) Experimental images for $\mathrm{LC}$ and $\mathrm{HC}$ material, respectively. (c, d) Simulated images for LC and HC material, respectively.

$p=0.0747)$, values $d_{3}=126 \mathrm{~nm}, p_{1}=0.547, p_{2}=0.41$ and $q_{3}=0.667$ were obtained. For the $\mathrm{HC}$ composite (with $p=0.1035)$, values $d_{3}=120 \mathrm{~nm}, p_{1}=0.48, p_{2}=0.461$ and $q_{3}=0.532$ were obtained.

This approach was used for several nanocomposites prepared in different conditions. An additional qualitative validation can be made by comparison between actual and simulated thin sections of materials, as shown in Figure 3.

In a following step, 3D simulations of the mesostructure of the materials were generated, as illustrated in Figure 4.

From the 3D simulations, it is possible to predict the effective (or macroscopic) permittivity of a composite, knowing the permittivity of individual components and the geometry. A code based on the Fast Fourier Transform [1] was used to numerically solve the electrostatics equations on the simulations of the mesostructure, following the approach developed in [8]. This code was validated for several models of composites with a known theoretical effective permittivity. In the present case, measured permittivities $\varepsilon_{1}=(3.5+0.2 \mathrm{i}) \varepsilon_{0}$ and $\varepsilon_{2}=(258+138 \mathrm{i}) \varepsilon_{0}$ were used for pure resin and carbonblack, respectively, estimated by fitting experimental data to an effective-medium analytical model in a wide range of compositions [3]. A total of 20 realizations of the random medium $\left(600 \times 600 \times 600 \mathrm{~nm}^{3}\right.$ over $128 \times 128 \times 128$ voxels) were used to estimate the effective permittivity with a $3 \%$ relative precision due to statistical fluctuations of the mesostructure. Values of $\varepsilon^{*} / \varepsilon_{0}=4.13+0.28 .0 \mathrm{i}$ and $\varepsilon^{*} / \varepsilon_{0}=4.37+0.32 \mathrm{i}$ were obtained for the LC and HC composites, respectively. These values are not consistent with

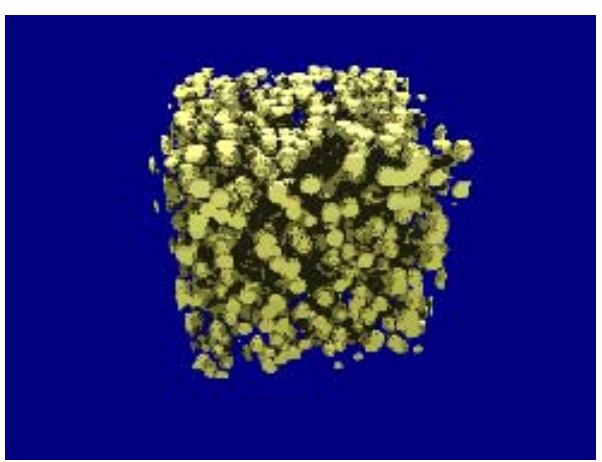

(a)

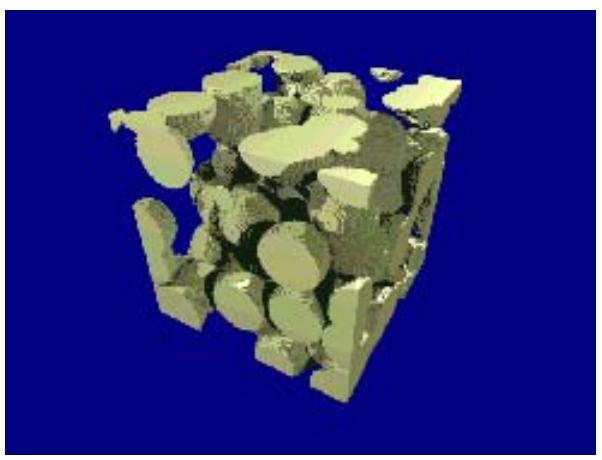

(b)

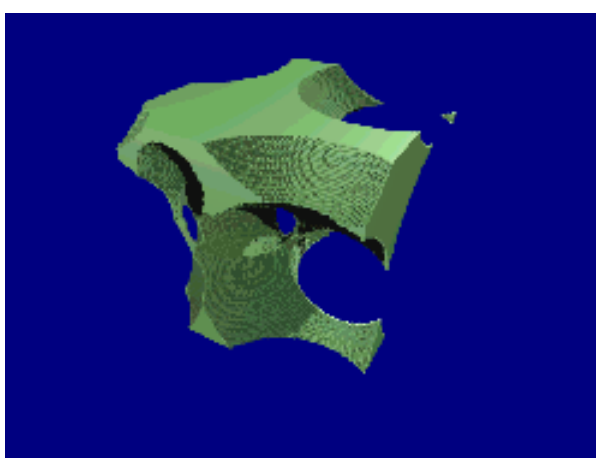

(c)

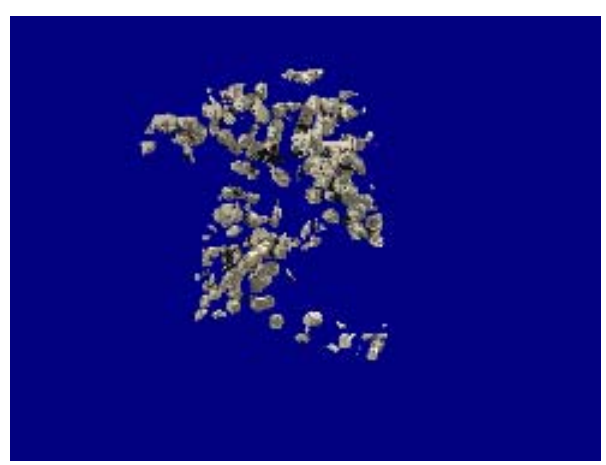

(d)

Fig. 4. 3D simulation of the mesostructure of a composite $\left(600 \times 600 \times 600 \mathrm{~nm}^{3}\right) .(\mathrm{a}, \mathrm{b}, \mathrm{c}, \mathrm{d})$ correspond to Figure $2(\mathrm{a}$, $\mathrm{b}, \mathrm{c}, \mathrm{e})$, respectively. 


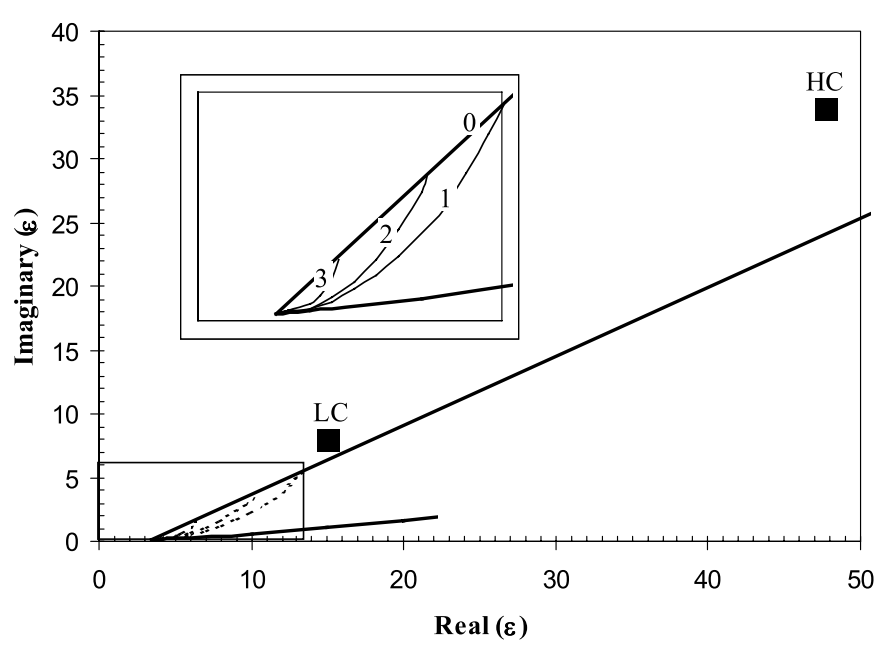

Fig. 5. Comparison of the measured permittivity for LC and HC composites (squares) to the bounds $(0,1,2,3)$ in the complex plane. The inset shows an enlarged view of the rectangular region on the bottom-left.

the measured permittivity given above, since they strongly underestimate their imaginary part.

Note that using a higher permittivity for the carbon black, such as $\varepsilon_{2}=(5526+6452 \mathrm{i}) \varepsilon_{0}$, as proposed in another study on similar composites [9], increases the effective permittivity by only about $5 \%$, since the carbon-black content is very low. In addition, the measured permittivity is outside of the zero-order BergmanMilton $[10,11]$ bounds depending only on the permittivities of the two components, which must be physically rejected. The bounds of orders 1,2 and 3, take into account namely the volume fractions, second and third order statistics of the medium (see Fig. 5).

Therefore it must be concluded that the permittivity of the resin in the composite has higher real and (mainly) imaginary parts, as compared to the pure resin used for the measurements. It can be assumed that this is the effect of a partial migration of carbon-black in the resin, as a result of an intense mixing during the preparation of the batch. This is investigated in the next parts of this paper.

\section{A hypothesis on material modification}

There is a possibility that the shear force introduced to overcome the cohesion force between carbon particles is strong enough to pull out weakly bonded (100) carbon planes from the onion-like structure. If this is the case, the carbon particles will have a tendency to get thinner without changing their intrinsic nature, and carbon planes will dissolve within the resin matrix. As a consequence, the electrical properties will be strongly affected, e.g. electrical conductivity will be drastically increased. This could explain the discrepancy between model and measurements described above.
There are two independent experimental approaches to test this hypothesis:

- A direct local physical measurement of a parameter that is affected by the enhancement of carbon chains/planes in the matrix: the most appropriate approach is an EELS analysis of the carbon edge with a nanometer size electron probe, or the calculation of the dielectric function from the low-loss region of EELS spectra;

- A direct observation of the dissolution of carbon planes in the matrix of the composite. Since the distances involved are in the nanometer scale, HRTEM must be used. However, these spatial frequencies will be hidden within a non-crystalline polymeric matrix, precluding typical Fourier space filtering, rotational average background subtraction methods [12], or template-matching pattern recognition approaches [13]. Structure modeling and image simulation [14] is also not useful in this case. Quantitative HRTEM image analysis must be carried out with a method that allows detecting very small periodic features within a non-periodic background. One can think of an approach that is sensitive to the "configuration" entropy of the pixel intensity distribution (which is thought to be higher in the pure resin than in the resin in the composite, which may contain some degree of order due to the presence of portions of carbon planes) and sensitive to the uniformity of the HRTEM images (which is thought to be higher in the resin containing carbon planes that restrict the grayscale variations).

Both routes have been followed in the present paper, although EELS experiments that were carried out must only be considered as a preliminary step (simple ELNES fingerprint approach).

\section{Nano-scale characterization}

\subsection{Electron energy loss spectroscopy}

Electron Energy Loss Spectroscopy (EELS) experiments were performed on a Philips CM20 microscope operating at $100 \mathrm{kV}$. The microscope is equipped with a Gatan PEELS spectrometer, the energy resolution and dispersion of which were $1 \mathrm{eV}$ and $0.1 \mathrm{eV}$ per channel, respectively. Spectra were acquired in the diffraction mode with a collection angle of $3.7 \mathrm{mrad}$. The convergence angle of the electron beam was $2.7 \mathrm{mrad}$. The spectra were corrected for noise and detector gain via the Gatan procedure. In order to allow inter comparison, the spectra were background-subtracted, plural scattering was removed by a Fourier-Ratio deconvolution procedure [15] and then their intensity was scaled to the maximum of the $285.5 \mathrm{eV}$ peak.

Several spectra were obtained on different regions of both pure resin and LC composite samples. On the latter, spectra were obtained either from regions where blackcarbon clusters are agglomerated within the resin or from regions far away from any visible carbon particle (composite between carbon clusters). The probe size was limited 


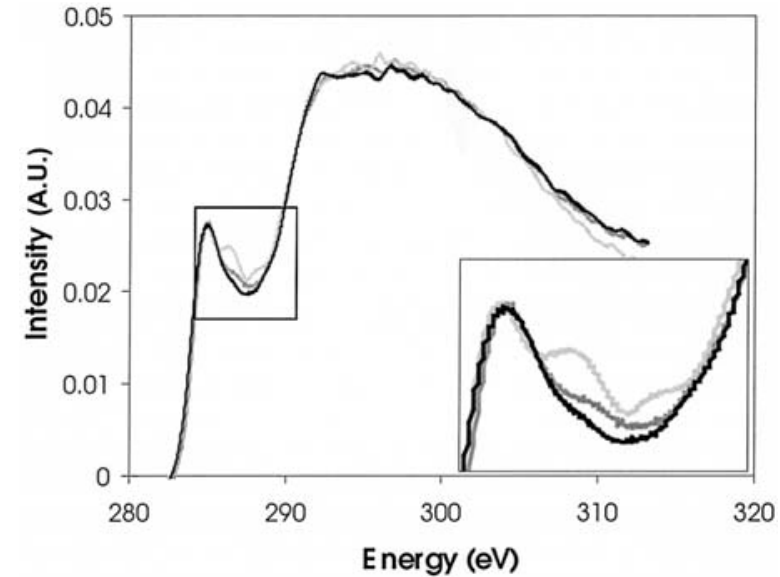

Fig. 6. EELS spectra for pure resin (light gray), LC composite between carbon clusters (dark gray) and LC composite on "carbon cluster" (black). The inset shows a magnified view of the framed region.

to $145 \mathrm{~nm}$, a compromise between a high intensity probe and a size that fitted the typical extent of carbon-black clusters.

Since it is supposed that (100) carbon planes are to be looked for, attention was focused on the carbon $K$-edge. Three characteristic spectra are shown superimposed in Figure 6 . The carbon $K$-edges are essentially characterized by two main features near 285.5 and $292.5 \mathrm{eV}$, which correspond respectively to transitions to $\pi^{*}$ and $\sigma^{*}$ states typically encountered in graphite-like compounds [16]. Looking at the [285-290] eV energy domain, a third signature appears after the $\pi^{*}$ peak, around $287 \mathrm{eV}$, the intensity of which decreases when going from pure resin to composite between carbon clusters. It nearly disappears for the case of the "carbon clusters". It is associated to $\sigma^{*}$ states corresponding specifically to the $\mathrm{C}-\mathrm{H}$ bonding in the resin matrix of the composite [17]. The fourth signature near $288.8 \mathrm{eV}$, which appears only in the case of the pure resin, can be attributed to $\pi^{*}(\mathrm{C}=\mathrm{O})$ states.

The structures extending from $285 \mathrm{eV}$ to $290 \mathrm{eV}$ for the resin in the composite are intermediate between those of the pure resin and those of the carbon cluster. Thus, it is thought that the signal for the resin in the composite is made partly of the contribution of pure resin and, in a significant proportion, of the contribution of a material similar to that of the carbon-black spheroids.

The question then is whether this dispersion of carbonblack material generalized through all the matrix resin? The measurement of bulk dielectric properties only indicates that percolation paths do exist. The answer can only come from a nano-scale texture analysis with appropriate discriminating parameters.

\subsection{Nano-scale texture analysis}

A typical image analysis sequence follows the steps of image acquisition, pre-processing, segmentation, postprocessing and measurement [18]. One of the most difficult steps is segmentation, where the particles or phases of interest must be discriminated from each other and from the background, to allow further measurement. The simplest approach to segmentation uses the pixel intensities as a discriminating characteristic between phases, choosing a threshold level through manual or automatic means [19]. Clearly, this is not possible for the case at hand as there are no clearly defined intensities or boundaries separating the two phases.

The proposed approach uses texture analysis based on the so-called Haralick Parameters [20], originally proposed for distinguishing different kinds of terrain in remote sensing applications. These parameters represent several statistics of the co-occurrence matrices of pixel intensities. Differently from typical occurrence statistics like the average or standard deviation of gray values, the co-occurrence matrices map the presence of pairs of pixel values in neighboring pixels at different orientations.

Haralick defined 14 parameters. Their full description is beyond the scope of this paper. However, a basic description of the co-occurrence matrices and some of the derived parameters will be useful. To simplify the discussion and adapt it to the application discussed herein, we shall consider a $N \times N$ pixels image, defining a spatial domain $L \times L$, where $L=\{1,2, \ldots, N\}$, and the grayscale values $G=\{1,2, \ldots, 255\}$.

The co-occurrence matrix for first $(d=1)$ horizontal $\left(\theta=0^{\circ}\right)$ neighbors located at positions $(k, l)$ and $(m, n)$ with gray values $i$ and $j$, respectively, is

$$
\begin{aligned}
& P\left(i, j, d=1, \theta=0^{\circ}\right)= \\
& \quad \#\left\{\begin{array}{l}
((k, l),(m, n)) \in(L \times L) \times(L \times L) \\
|k-m|=0,|l-n|=1, G(k, l)=i, G(m, n)=j
\end{array}\right\}
\end{aligned}
$$

where \# denotes the number of elements in the set. Thus, every pair of horizontal first neighbors is checked for the co-occurrence of gray values $i$ and $j$. The total number of such pairs, normalized by the total number of entries $R$ in the matrix $\left(R=G_{\max } \times G_{\max }\right)$, corresponds to entry $(i, j)$ in the matrix. It corresponds to the local bivariate distribution of the pair of gray levels observed in two neighboring points.

Normally the first neighbors in an 8-neighborhood are considered, but larger $d$ values can also be used. It is also common to consider the orientation average for $\theta=0,45,90$ and $135^{\circ}$, if directional characteristics are not important in the analyzed image. This is the approach followed in the present work.

Based on $P_{i j}$, several parameters can be defined. The most relevant to our discussion are

$$
\begin{aligned}
\text { Uniformity } & =\sum_{i} \sum_{j} P_{i j}^{2} \\
\text { Contrast } & =\sum_{n=0}^{G_{\max }-1} n^{2}\left\{\sum_{\substack{i=1 \\
|i-j|=n}}^{G_{\max }} \sum_{j=1}^{G_{\max }} P_{i j}\right. \\
\text { Entropy } & =-\sum_{i} \sum_{j} P_{i j} \log \left(P_{i j}\right) .
\end{aligned}
$$


Note that the Contrast parameter is equivalent to the variogram used in Geostatistics [7], estimated at distance $d$. These parameters were selected after careful analysis of the first 11 parameters, provided by the employed image analysis software [21]. Some of the parameters are correlated [20] to each other and their simultaneous use does not contribute to discrimination. Moreover, it is not always easy to derive a physical meaning for many of them. The analysis was thus restricted to parameters that were found to be uncorrelated, discriminating and physically meaningful.

One should expect a more disordered nano-structure to have lower Uniformity and higher Entropy at a local level. The presence of spatially organized carbon layers should lead to higher niformity, increasing the organization of the image, thus reducing its Entropy. Similarly, carbon layers should reduce the local contrast, as a relatively large number of pixels would have very similar intensities along atomic planes.

Thus, pure resin and LC composite samples were studied. For the composite sample, only regions away from carbon clusters were analyzed. The main steps of the procedure are described in the following.

\subsubsection{Image acquisition}

In order to start a nano-scale contrast analysis with details as fine as single (100) carbon planes, one must record images at high magnification and at high resolution in the TEM phase contrast mode. HRTEM images were obtained in the same TEM as for the mesoscopic analysis. Great care was taken to record images at a defocus as close as possible to Scherzer defocus, both to approach optimal performance of the microscope $(0.24 \mathrm{~nm}$ point-topoint resolution) as to allow the comparison of the spatial frequencies from one image to another. This was systematically checked through numerical diffractograms.

The images were captured on film and subsequently digitized with a high-resolution drum scanner at $2048 \mathrm{dpi}$, corresponding to $0.023 \mathrm{~nm} /$ pixel. Figure 7 shows HRTEM images of the pure resin (top), of the LC composite material in a region between carbon clusters (middle) and of the LC composite where two carbon particles can be seen (bottom).

Even though the two images on the top and middle rows of Figure 7 look very similar, close visual inspection reveals differences, represented by small regions that seem to correspond to small carbon planes in the LC composite image.

The question is whether these subtle changes between pure resin samples and composite samples away from carbon clusters, barely visible in HRTEM images, can be quantified.

\subsubsection{Pre-processing}

All images were pre-processed to equalize average brightness and eliminate the influence of microscope illumination and/or film processing variations.
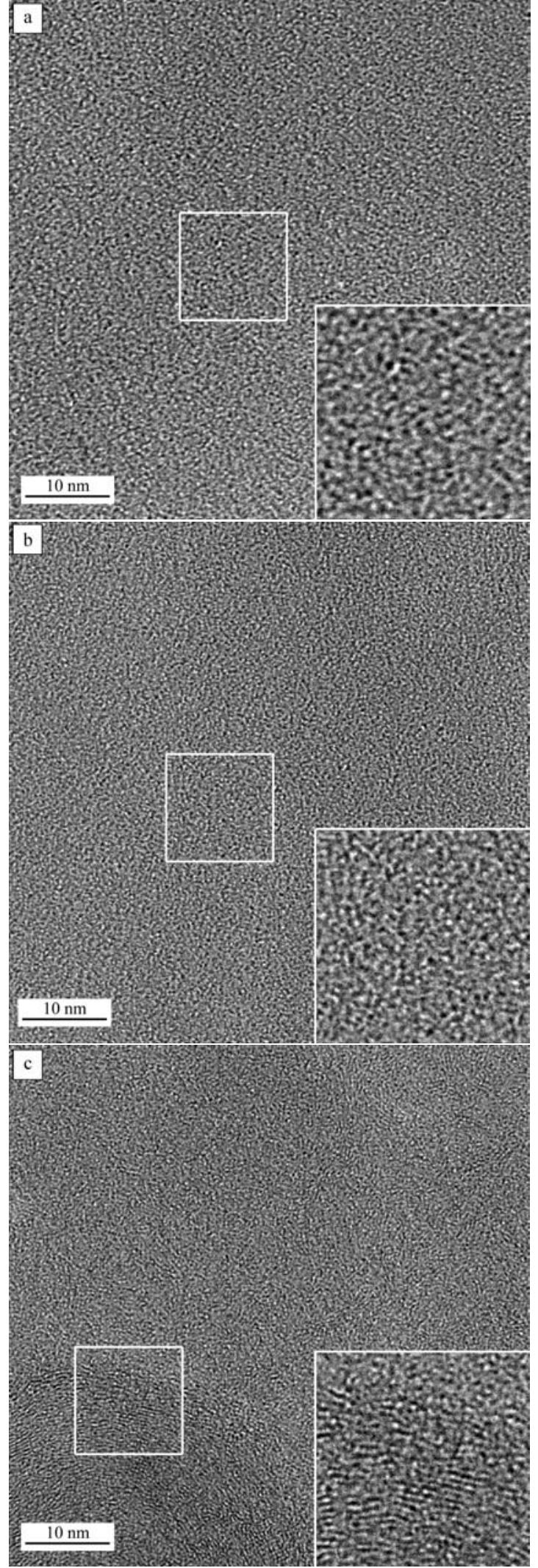

Fig. 7. HRTEM images of (a) pure resin, (b) LC composite material away from carbon clusters, and (c) LC composite material with carbon particles. The insets show $2 \times$-magnified views of the regions marked by the white squares. 

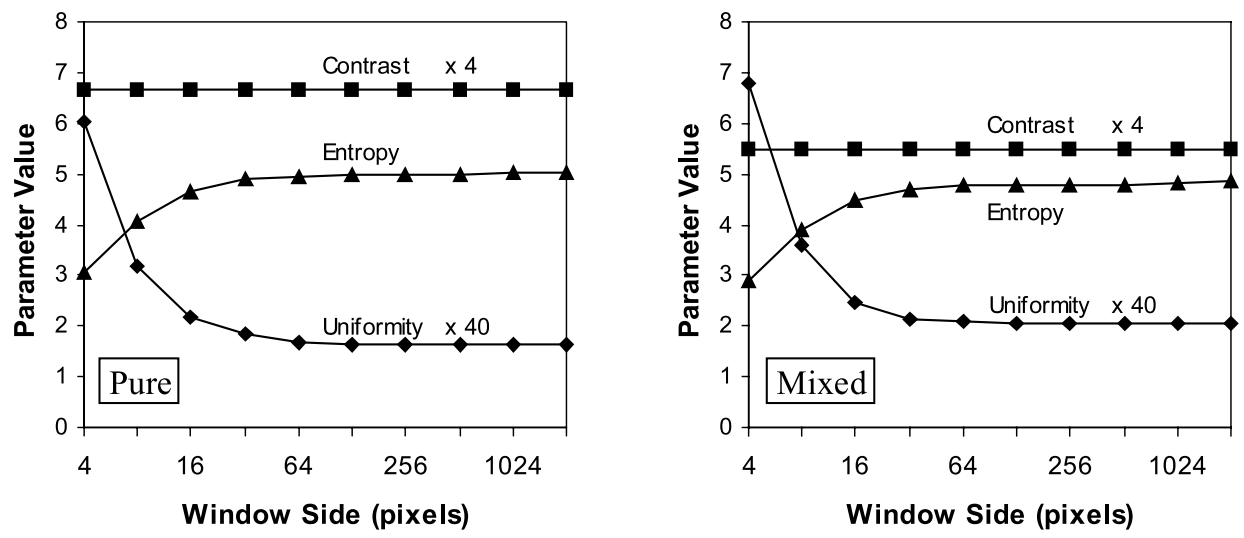

Fig. 8. Dependence of Uniformity, Contrast, and Entropy on the window size for the pure resin and LC composite (mixed) samples away from carbon clusters. Results correspond to the average of 9 images of each kind (notice the magnified scales for Contrast and Uniformity).
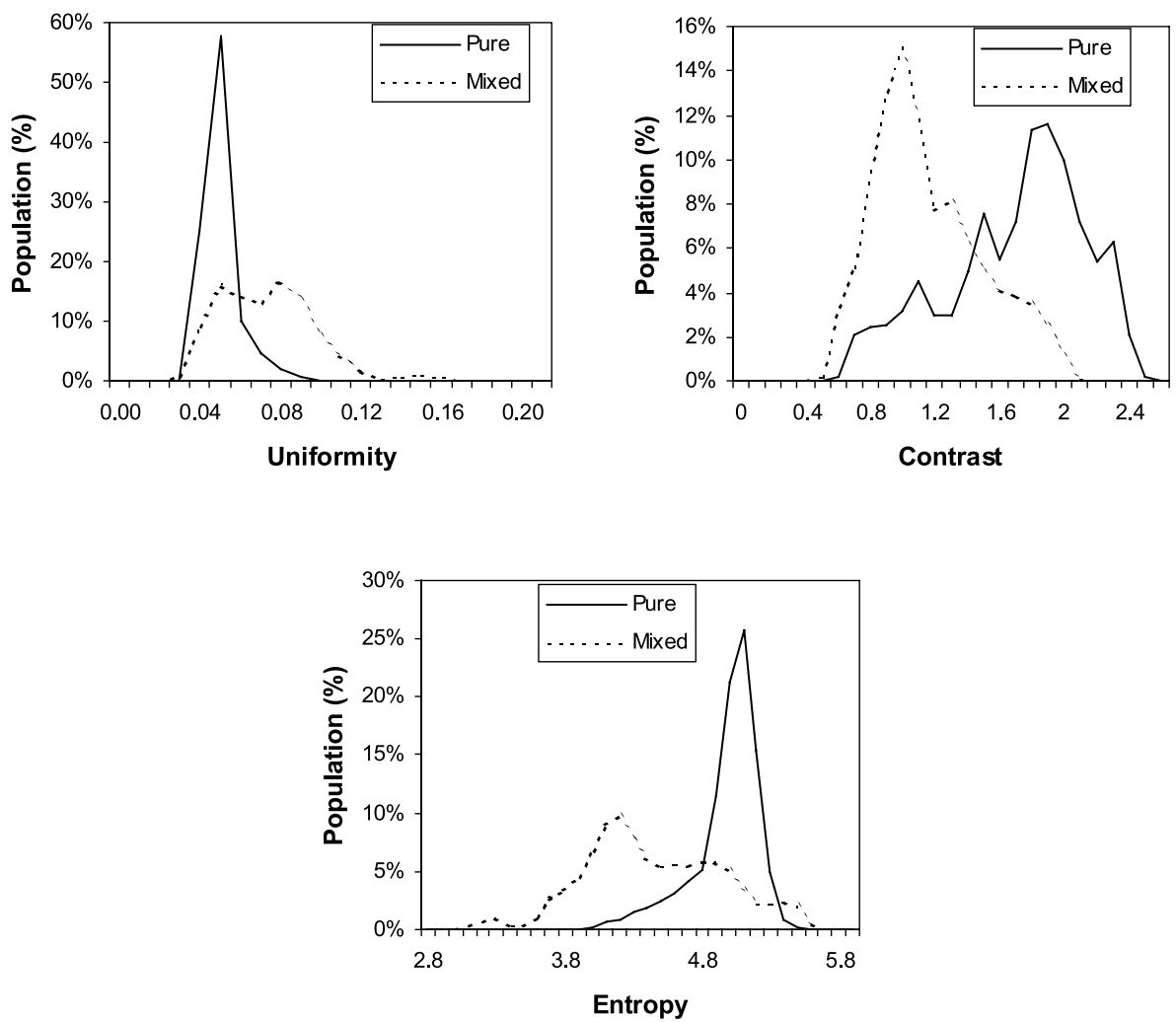

Fig. 9. Histograms for Uniformity, Contrast, and Entropy for pure resin and LC composite (mixed) samples away from carbon clusters.

\subsubsection{Window size influence}

Each image was divided into equal-sized adjoining square windows. Window sizes ranged from $4 \times 4$ to $2048 \times$ 2048 pixels, the total size of the image. A typical dependence of the Haralick parameters on window size was found, as shown in Figure 8. The behavior is very similar for both kinds of samples. Uniformity decreases and Entropy increases with increasing window size while Contrast is not affected. The asymptotic values are reached for windows between $64 \times 64$ and $128 \times 128$ pixels, corresponding to $1.92 \times 1.92$ and $3.84 \times 3.84 \mathrm{~nm}$. These values correspond to between 5 and 10 atomic planes of carbon.

All further analyses were done with $100 \times 100$ pixels windows. This size is in the asymptotic region and gives a total of $400(20 \times 20)$ windows in each image, allowing for a small guard frame to eliminate possible edge effects.

\subsubsection{Statistical analysis}

Nine different images of the pure resin were available, providing a total of 3600 analyzed windows. As a wider 


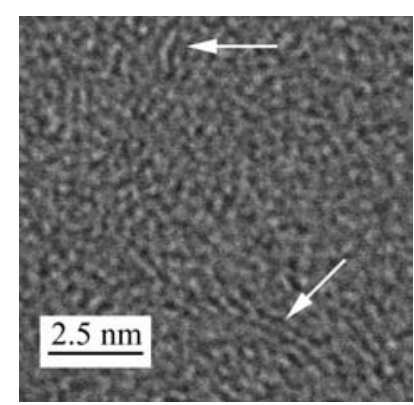

Fig. 10. HRTEM image of the LC composite sample away from carbon clusters. The arrows indicate small, organized layers, probably of carbon.

variation of properties was expected for the composite material, a larger number of 44 images, for a total of 17600 windows, was used.

Histograms for the 3 main Haralick parameters were then obtained for both materials. The results are shown in Figure 9.

The histograms clearly show differences between pure resin and LC composite (mixed) samples at a nanostructural level. The main characteristics that can be derived from these plots are:

(a) The pure resin images show less variation in texture, as shown by the narrower histograms for Uniformity and Entropy.

(b) Part of the composite population has a higher Uniformity than the pure resin (peaks at 0.09 and 0.15 in the Uniformity histogram). On the other hand, part of the population has very similar Uniformity values (peak at 0.04 ) to the pure resin. This can indicate a separation between carbon-full and carbon-free regions in the composite samples.

(c) The Contrast histogram shows wide variations for both pure resin and composite. Nevertheless, the composite shows overall smaller Contrast values. As mentioned above, this can be explained by the presence of small carbon layers that would create regions of approximately constant pixel intensity values along carbon planes. Figure 10 shows an example.

(d) The Entropy is clearly lower for the composite, meaning the material becomes more organized with the presence of the carbon layers. Nevertheless, it shows an Entropy peak at value 5, roughly coinciding with the pure resin peak. This is consistent with the discussion in item $b$.

\section{Conclusions}

\subsection{About the material}

The composite fabrication process is energetic enough to substantially erode the carbon-black particles so that a significant amount of carbon is dispersed within the resin matrix. This finely dispersed material, which has more or less the properties of carbon-black (maybe a higher electron conductivity since surface plasmons may appear as a consequence), renders the matrix much more conductive than it was supposed to be.

The morphology of this material strongly enhances the capability of the composite to attain the percolation threshold for conduction at constant concentration, as compared to a composite with only simple carbon-black clusters. This is clearly visible when one compares the bulk permittivity measurements to the prediction for the LC and $\mathrm{HC}$ materials.

Even if the material is not what it was supposed to be, the process allows the achievement of a certain range of properties at a lower carbon-black cost. One can think of tailoring the dielectric properties of such a material by the process/mixing/eroding time rather than by playing on the carbon-black concentration, which can quickly reach a limit due to viscosity/wettability problems. As the composite is to be used as a paint, and thus must exhibit a viscosity low enough to allow the deposition of layers a few tens of micrometers thick, a high concentration of carbonblack would strongly impair the fluidity of the material.

\subsection{About the methodology}

To fully characterize the material, a whole set of experiments and numerical tools were necessary, the complementary spatial resolution of which precisely allowed probing the various microstructural coherence lengths of the composite.

As both the mesoscopic random set morphological modeling and the bulk permittivity measurements are deemed reliable, the contradiction in their results must mean that the input data for the model (specifically the dielectric property of the resin in the composite) was wrong. It is thus essential that these models take into account local properties when there are questions about the stability of the different phases during processing.

The Haralick parameter approach has been found to be as relevant for nano-scale analysis of materials as it has originally been for discriminating the nature of grounds in remote sensing. In that sense, it appears to be complementary to the mesoscopic random set approach to which it is nevertheless somehow related. It should be noted that variations in focus for High Resolution Transmission Electron Microscopy imaging will likely affect the Haralick values although the exact influence is hard to foresee as it would be an intricate relation between the distance between pairs of pixels, the window size and the Contrast Transfer Function of the Microscope (CTF). Astigmatism would also affect the results but its effects are averaged out in the present analysis, because the average of the four main directions was taken. It is also reasonable to expect some directional characteristics in regions of the matrix where carbon planes are present. In this sense, the pure resin should be more isotropic that the composite matrix but, as the orientation of carbon planes is very likely random, this effect would be hard to detect unless specific regions of the images, with specific orientations of carbon planes, were separately analysed. Again, the use 
of the directional average eliminates this influence in the present analysis.

The EELS analysis was consistent with the nano-scale texture analysis and confirmed the presence of carbon dispersed in the resin matrix. However, the exact signification of the $\pi$ and $\sigma$ fine structure requires further investigation. More research is needed to get measurements of the local dielectric permittivity on a micro or meso scale. This would be useful for the prediction of macroscopic properties from models describing the mesostructure of composite materials.

S.P. wishes to thank École des Mines de Paris for a yearly position as visiting professor (1999-2002) and acknowledges the support of the Brazilian agencies $\mathrm{CNPq}$ and FINEP/MCT, through the RECOPE and Pronex projects. A.D. thanks the French Délégation Générale à l'Armement for a grant that allowed him to prepare and present a Ph.D. thesis in 2001. All the authors wish to thank Philippe Masclet (DGA) for his constant support, Gérard Piau (EADS) for his help and for the preparation of the materials, and both for fruitful and stimulating discussions.

\section{References}

1. A. Delarue, Ph.D. thesis, Paris School of Mines, 2001

2. D. Jeulin, A. Le Coënt, in Proceedings of CMDS8 International Symposium, Varna, Bulgaria, 1995, edited by K.Z. Markov (World Scientific Publishing Company, 1996), p. 199

3. C. Marchand, Ph.D. thesis, Institut National Polytechnique de Lorraine, (1998)
4. A. Thorel, L. Normand, B. Delcroix, C. Echer, P. Cheminant-Coatanlem, Y. Montardi, in Ceramic Interfaces 2, edited by H.-I. Yoo, S.-J.L. Kang (IOM Communications Ltd, 2001), p. 397

5. L.L. Savary, D. Jeulin, A. Thorel, Acta Stereol. 18, 297 (1999)

6. G. Matheron, in Éléments pour une Théorie des Milieux Poreux (Paris, 1967)

7. G. Matheron, in Random Sets and Integral Geometry (J. Wiley, New York, 1975)

8. D.J. Eyre, G.W. Milton, Eur. Phys. J. AP 6, 41 (1999)

9. D.S. McLachlan, A. Priou, J. Electromagn. Waves Appl. 6, 1099 (1992)

10. D.J. Bergman, Phys. Rep. 43 C, 377 (1978)

11. G.W. Milton, Appl. Phys. Lett. 37, 300 (1980)

12. R. Kilaas, J. Microsc. 190, 45 (1998)

13. S. Paciornik, R. Kilaas, J. Turner, U. Dahmen, Ultramicroscopy 62, 15 (1996)

14. R. Kilaas, in Proceedings of the Microscopy Society of America 51st annual meeting (1993), p. 542

15. R.F. Egerton, in Electron Energy Loss Spectroscopy in the Electron Microscope, 2nd edn. (Plenum Press, New York, 1996)

16. R.D. Leapman, P.L. Fejes, J. Silcox, Phys. Rev. B 28, 2361 (1983)

17. K. Varlot, J.M. Martin, C. Quet, Y. Kihn, Ultramicroscopy 68, 123 (1997)

18. P.R.M. Vieira, S. Paciornik, Mater. Charact. 47, 219 (2002)

19. J.C. Russ, in Computer Assisted Microscopy (Plenum Press, New York, 1991)

20. R.M. Haralick, K. Shanmugan, I. Dinstein, IEEE Trans. Syst. Man Cybern. SMC-3, 610 (1973)

21. KS400, Version 3, Carl Zeiss Vision, (2000) 